\title{
Fractal model for dielectric relaxation in deuteron pseudospin glass DRADP
}

\section{Authors: V. Hugo Schmidt and Darin Arbogast}

This is a postprint of an article that originally appeared in AIP Conference Proceedings on August 2002. The final version can be found at https://dx.doi.org/10.1063/1.1499555.

V. Hugo Schmidt and Darin Arbogast, "Fractal model for dielectric relaxation in deuteron pseudospin glass DRADP," Fundamental Physics of Ferroelectrics 2002, Washington, DC, AIP Conference Proceedings 626, 81-88 (2002). doi: 10.1063/1.1499555

Made available through Montana State University's ScholarWorks

scholarworks. montana.edu 


\title{
Fractal Model for Dielectric Relaxation in Deuteron Pseudospin Glass DRADP
}

\author{
V. Hugo Schmidt and Darin Arbogast* \\ Physics Department, Montana State University, Bozeman, MT 59717 \\ *Now at Boeing Airplane Co., Seattle, WA
}

\begin{abstract}
Proton and deuteron glasses such as $\mathrm{Rb}_{1-\mathrm{x}}\left(\mathrm{ND}_{4}\right)_{\mathrm{x}} \mathrm{D}_{2} \mathrm{PO}_{4}$ (DRADP) are ideal systems for investigating dynamics of spin-glass-type systems because the basic mechanism for their dynamics is well understood. This mechanism consists of three processes; creation, effective diffusion, and annihilation of $\mathrm{DPO}_{4}$ and $\mathrm{D}_{3} \mathrm{PO}_{4}$ "Takagi groups." Each process involves a deuteron transfer from one side of an $\mathrm{O}-\mathrm{D} \cdots \mathrm{O}$ bond to the other. The effective diffusion changes the configurational energies of the $\mathrm{D}_{2} \mathrm{PO}_{4}$ "Slater groups" traversed by the Takagi groups. Each diffusion step changes this energy by a random amount with magnitude of order $\varepsilon_{\mathrm{d}}$. This $\varepsilon_{\mathrm{d}}$ is comparable to the basic energy $\varepsilon_{0}$ of the Slater model for $\mathrm{RbD}_{2} \mathrm{PO}_{4}$, and considerably smaller than the Takagi $\mathrm{DPO}_{4}-\mathrm{D}_{3} \mathrm{PO}_{4}$ pair creation energy $2 \varepsilon_{\mathrm{c}}$. The Takagi group diffusion path between creation and annihilation on average does not change the configurational energy. Thus the energy landscape along the path has an unbiased fractal nature, with small energy barriers superimposed on larger ones. The Takagi group diffusion path has side branches that are retraced, and loops of six or more steps. We approximate this path by a deterministic fractal path, having shorter side branches superimposed on longer ones, attached to a trunk running from the creation to the annihilation site. The longest dielectric relaxation time constant is governed by the Boltzmann factor for the highest barrier on the entire trunk. Shorter time constants correspond to the highest barriers on shorter trunk or branch segments. The relaxation strength distribution over these time constants depends on the basic fractal path unit, namely the number $\mathrm{m}$ of forward steps per side step. It depends also on temperature $\mathrm{T}$ and on $\varepsilon_{\mathrm{c}}$ and $\varepsilon_{\mathrm{d}}$. This model predicts with good qualitative accuracy the $\mathrm{T}$ and $\mathrm{f}$ (frequency) dependences of the real $\left(\varepsilon^{\prime}\right)$ and imaginary ( $\varepsilon$ ") parts of the dielectric permittivity measured by Courtens ${ }^{1}$ in $x=0.62$ DRADP over wide $\mathrm{T}$ and $\mathrm{f}$ ranges. No adjustable parameters were used except for $\mathrm{m}$. The static and high- $\mathrm{f}$ permittivities $\varepsilon_{\mathrm{s}}$ and $\varepsilon_{\infty}$ were chosen to fit Courtens' data, while $\varepsilon_{\mathrm{c}}$ and $\varepsilon_{\mathrm{d}}$ were set at the values $\varepsilon_{\mathrm{c}} / \mathrm{k}=940 \mathrm{~K}$ and $\varepsilon_{\mathrm{d}} / \mathrm{k}=140 \mathrm{~K}$ chosen by the Blinc/Kind groups to fit DRADP NMR data. ${ }^{2}$ This consideration of diffusion path topology and prediction of dielectric permittivity are major extensions of our previous work. ${ }^{3}$
\end{abstract}

\section{INTRODUCTION}

The dynamics of glassy behavior is a fascinating subject, whose development is hindered because in most glassy systems there is no well-defined dynamic process. Proton and deuteron glasses do have a well-defined dynamic process, and so a more

CP626, Fundamental Physics of Ferroelectrics 2002, edited by R. E. Cohen (C) 2002 American Institute of Physics 0-7354-0079-2/02/\$19.00 
precise model can be developed for their dynamics. We develop such a model in this work, and show how it predicts the ac dielectric permittivity behavior quite accurately over a wide temperature and frequency range.

The model is based on treating the crystal as a bound charge semiconductor, that exhibits the usual semiconductor processes of carrier creation, diffusion, and annihilation. The diffusion is dominated by a fractal distribution of diffusion barriers. A fruitful approximation is the assumption of a deterministic fractal geometry of the diffusion path. We develop the relation of this fractal path to the dc permittivity. We find that the wide distribution of time constants in the ac permittivity is related to the distribution of fractal generations. The model with discrete fractal generations is too coarse to be useful, so we generalize it to an integral formulation for dielectric permittivity. This integral formulation gives good agreement with experiment over a wide temperature and frequency range. We now treat each of these model features in turn.

\section{BOUND CHARGE SEMICONDUCTOR DESCRIPTION}

This description applies to H-bonded crystals with H's in off-center bond positions. The bonds should be symmetric enough in the time average to permit intrabond hydrogen transfer. This requirement rules out participation of $\mathrm{N}-\mathrm{H} . . . \mathrm{O}$ and other intrinsically asymmetric $\mathrm{H}$-bonds in this particular dynamic process. We will now show how the bound charge semiconductor description applies to the deuteron glass $\mathrm{Rb}_{1-\mathrm{x}}\left(\mathrm{ND}_{4}\right)_{\mathrm{x}} \mathrm{D}_{2} \mathrm{PO}_{4}$ (DRADP) $(\mathrm{x}=0.62)$.

DRADP is a mixed crystal whose parent crystals $\mathrm{RbD}_{2} \mathrm{PO}_{4}$ and $\mathrm{ND}_{4} \mathrm{D}_{2} \mathrm{PO}_{4}$ have ferroelectric (FE) and antiferroelectric (AFE) transitions respectively. The quenched randomness of the $\mathrm{Rb}^{+}$and $\mathrm{ND}_{4}{ }^{+}$ion placement results in frustrated FE and AFE interactions, so for a wide range of $\mathrm{x}$, including $\mathrm{x}=0.62$, DRADP has no transition. Instead, it gradually changes from a normal paraelectric (PE) to a dipolar glass or pseudospin glass as temperature decreases.

The pseudospin (in analogy with spin in magnetic spin glasses) is 1 or -1 for the two deuteron positions in its O-D $\cdots \mathrm{O}$ bond. The deuteron cannot leave the bond, so it is a bound charge.

If these deuterons are bound charges, how can we call the crystal a bound charge semiconductor? The answer lies in the Pauling "ice rules" that apply also to DRADP and many other H-bonded crystals. These rules are: One hydrogen in each hydrogen bond; Two hydrogens close to a given $\mathrm{PO}_{4}$ in the four bonds connecting that phosphate ion to its neighbors. These rules were the basis of the Slater ${ }^{4}$ model of $\mathrm{KH}_{2} \mathrm{PO}_{4}(\mathrm{KDP})$, and allow only $\mathrm{D}_{2} \mathrm{PO}_{4}$ configurations of phosphate ions and their close deuterons. Even though these rules allow randomness in the hydrogen configurations in the PE state (in analogy to the randomness found in ice), they allow no deuteron intrabond motion, and hence no semiconductivity.

The semiconductivity arises when the second ice rule is broken, following a suggestion by Takagi. ${ }^{5}$ Breaking this rule allows creation or annihilation of charge 
carrier pairs by means of the following reaction that involves only intrabond deuteron transfer:

$$
\mathrm{D}_{2} \mathrm{PO}_{4}+\mathrm{D}_{2} \mathrm{PO}_{4} \leftrightarrow \mathrm{DPO}_{4}+\mathrm{D}_{3} \mathrm{PO}_{4}
$$

Such a carrier pair is created at the cost of a "gap" energy $2 \varepsilon_{\mathrm{c}}$. These $\mathrm{DPO}_{4}$ and $\mathrm{D}_{3} \mathrm{PO}_{4}$ "Takagi groups" rather than the individual deuterons are called the carriers because they in effect diffuse through the crystal by means of the following reactions that involve only intrabond deuteron transfer:

$$
\mathrm{DPO}_{4}+\mathrm{D}_{2} \mathrm{PO}_{4} \rightarrow \mathrm{D}_{2} \mathrm{PO}_{4}+\mathrm{DPO}_{4} \quad \mathrm{D}_{3} \mathrm{PO}_{4}+\mathrm{D}_{2} \mathrm{PO}_{4} \rightarrow \mathrm{D}_{2} \mathrm{PO}_{4}+\mathrm{D}_{3} \mathrm{PO}_{4}
$$

Carrier creation, diffusion, and annihilation are characteristic of semiconductors. It only remains to show that these Takagi group carriers actually carry charge, in order to justify the bound charge semiconductor designation.

To find this charge, which is actually a charge tensor, consider the process of polarization reversal from $-\mathbf{p}$ to $\mathbf{p}$ of a fully polarized unit cell of volume $a^{2} c$. Its dipole moment changes by $2 \mathrm{P}_{\mathrm{s}} a^{2} c$ because of 8 intrabond transfers, because there are $Z=4$ formula units per unit cell, and 2 "acid" $O-D \cdots O$ deuterons per unit cell. Here, $P_{s}$ is approximately the spontaneous polarization of the FE parent crystal, DRDP. Each transfer moves a $\mathrm{DPO}_{4}$ carrier a distance $-c / 4$ or a $\mathrm{D}_{3} \mathrm{PO}_{4}$ carrier a distance $c / 4$, along the $c$ axis. Dividing the moment change per transfer by the carrier $c$-axis displacement per transfer gives $\mathrm{P}_{\mathrm{s}} a^{2}$ as the $\mathrm{q}_{\mathrm{cc}}$ element of the diagonalized charge tensor for the $\mathrm{D}_{3} \mathrm{PO}_{4}$ carrier, and $-\mathrm{P}_{\mathrm{s}} a^{2}$ for the $\mathrm{DPO}_{4}$ carrier. The size of the two equal $\mathrm{q}_{\mathrm{aa}}$ elements cannot be determined exactly because there is no ordered state with polarization perpendicular to $c$.

\section{CARRIER CREATION, DIFFUSION, AND ANNIHILATION}

We now examine details of these three processes. They involve only intrabond transfer and thus cannot contribute to dc conduction. The dc conduction mechanism has negligible effect at low temperatures where deuteron glass phenomena occur.

The rate for the carrier creation that occurs via the reaction of Eq. (1) is

$$
\mathrm{dn}_{\mathrm{c}} / \mathrm{dt}=4 \mathrm{~N}(\mathrm{kT} / \mathrm{h}) \exp \left(-2 \varepsilon_{\mathrm{c}} / \mathrm{kT}\right) \text {, }
$$

where $\mathrm{N}$ is the density of formula units, and $\mathrm{kT} / \mathrm{h}$ is the attempt rate $\mathrm{v}$, based on $\mathrm{h} v=\mathrm{kT}$. We use this relation rather than allowing $v$ to be an adjustable parameter.

The carrier diffusion via the reaction of Eq. (2) occurs on a 3-dimensional $\mathrm{H}$-bonded network in which topologically the $\mathrm{H}$-bonds correspond to the $\mathrm{C}-\mathrm{C}$ bonds in the diamond lattice. Each bond acts as a "diode" that allows $\mathrm{D}_{3} \mathrm{PO}_{4}$ traverse in one direction, and $\mathrm{DPO}_{4}$ traverse in the other. Once the bond is traversed, the diode is reset in the opposite direction. Each carrier move is to one of two new positions or to its previous site, so the possible diffusion paths locally correspond to a double- 
branching Cayley tree or Bethe lattice. The shortest-scale breakdown of the Cayley tree analogy occurs for loops of $6 \mathrm{H}$-bonds, but traversal of such a loop requires that the "diodes" of all 6 bonds be set in the correct direction.

The carrier annihilation rate is

$$
\mathrm{dn}_{\mathrm{a}} / \mathrm{dt}=-\mathrm{n} / \tau_{1}, \quad\left(\tau_{1} \text { is carrier lifetime }\right) . \quad \text { In equilibrium, } \quad \mathrm{dn}_{\mathrm{c}} / \mathrm{dt}+\mathrm{dn}_{\mathrm{a}} \mathrm{dt}=0,
$$

so from Eqs. (3) and (4), the equilibrium carrier concentration is

$$
\mathrm{n}=4 \mathrm{~N} \tau_{1}(\mathrm{kT} / \mathrm{h}) \exp \left(-2 \varepsilon_{\mathrm{c}} / \mathrm{kT}\right)
$$

\section{FRACTAL DISTRIBUTION OF DIFFUSION BARRIERS}

In $\mathrm{RbD}_{2} \mathrm{PO}_{4}$ (DRDP), the "Slater" $\mathrm{D}_{2} \mathrm{PO}_{4}$ groups according to the Slater model have "configurational" energies 0 or $\varepsilon_{0}$, and just above $T_{c}$ either energy is equally likely. Thus, in 4 diffusion steps, approximately 2 will not change the configurational energy, and approximately one each will change the energy by $\varepsilon_{0}$ or $-\varepsilon_{0}$. Equality of probability of upward and downward steps is required in equilibrium to conserve configurational energy in the time average.

In DRADP, an antiferroelectric configurational energy also comes into play, ${ }^{6}$ but one can still assume that on the net diffusion path, an upward energy step mean height $\varepsilon_{\mathrm{d}}$ near $\varepsilon_{0}$ occurs approximately every 4 diffusion steps.

The configurational energy changes along the actual net diffusion path thus constitute an unbiased random walk in energy space. The highest barrier encountered in $M$ net steps will be of order $(M / 4)^{1 / 2} \varepsilon_{d}$, and there will be lower barriers, and superimposed on them still lower barriers, constituting a fractal energy landscape. However, the typical available diffusion path will not be unbiased, but will tend to go uphill in energy. It is the Boltzmann factors for the three available choices for each step that keeps the actual path level on the average. As temperature decreases, the proportion of lower-energy Slater groups increases, and the average available path becomes steeper uphill. This means that the possible paths that are level on the average become sparser as temperature decreases. The proportion of lower-energy Slater groups cannot approach unity in a disordered H-bond configuration, because the entropy will first go to zero at some temperature $\mathrm{T}_{\mathrm{e}}$, according to our previous model. ${ }^{6}$

\section{FRACTAL GEOMETRY OF DIFFUSION PATH}

The Takagi group diffusion has a doubly fractal nature. We saw above that the configurational energy landscape of the diffusion path is fractal. The spatial diffusion path is qualitatively a deterministic fractal path, with smaller side branches extending out from larger branches, etc., until the main trunk leading from creation to annihilation is reached. A deterministic fractal path is assumed to allow closed-form mathematical analysis. This deterministic fractal path differs from the actual path in two ways. First, the branches are random, not deterministic. Second, the occurrence 
of loops is not taken into account. Still, this deterministic path and its associated dynamics provide a good qualitative fit to the observed phenomena.

How are these fractal paths described? An arrow $\rightarrow$ indicates a Takagi group transfer from one phosphate location to a nearest neighbor location and represents the $0^{\text {th }}$ generation path. An infinite number of choices exists for the first generation path. Succeeding generation paths are determined once this choice is made. The two choices we used so far are the $\mathrm{m}=2$ and the $\mathrm{m}=41^{\mathrm{st}}$-generation 4 -step sequences

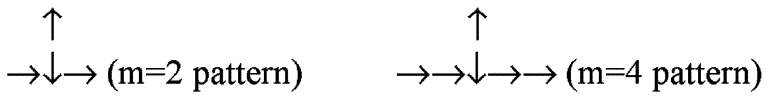

that consists of $2(\mathrm{~m}=2)$ or $4(\mathrm{~m}=4)$ forward steps separated by a side step and its return step. A $2^{\text {nd }}$-generation path segment is formed by replacing each of the arrows in either pattern in (6) by the entire corresponding pattern. A $3^{\text {rd }}$-generation path segment consists in replacing each arrow in the $2^{\text {nd }}$-generation path by the corresponding pattern in (6), etc., so each succeeding generation has a longer trunk and a more complex set of non-looping branches than the previous one.

Other possible basic patterns could have an odd number of main trunk steps, longer side steps, more than one side step, etc. By examining the chosen patterns in (6), we see that they obey the following relations for a path of $g$ generations:

Net path length $M=m^{g}$,

Number of possible annihilation sites visited is $(m+1)^{g}$,

Total number of steps taken is $(\mathrm{m}+2)^{\mathrm{g}}$.

\section{RELATION OF FRACTAL PATH TO DC PERMITTIVITY}

Each diffusion step in a field $\mathrm{E}$ contributes to the sample dipole moment. When $\mathrm{E}$ is turned on, initially the carriers visit new sites at a given rate. The new sites can hold annihilation partners, so when all new sites are visited, the annihilation partners will be found. The time required for this to occur is the carrier lifetime $\tau_{1}$. Each carrier has traveled a distance $\mathrm{m}^{\mathrm{g}}$ steps along the main trunk by this time, so the maximum barrier it has encountered is typically $\left(\mathrm{m}^{\mathrm{g}} / 4\right)^{1 / 2} \varepsilon_{\mathrm{d}}=\mathrm{m}^{\mathrm{g} / 2} \varepsilon_{\mathrm{d}} / 2$. At low temperature the maximum barrier will dominate the carrier lifetime, so it is given approximately by

$$
\tau_{\mathrm{l}}=(\mathrm{h} / \mathrm{kT}) \exp \left(\mathrm{m}^{\mathrm{g} / 2} \varepsilon_{\mathrm{d}} / 2 \mathrm{kT}\right) .
$$

The number of sites visited on this path from creation to annihilation is $(\mathrm{m}+1)^{\mathrm{g}}$, from Eq. (8). Each site has probability $\mathrm{n} / \mathrm{M}$ of hosting an annihilation partner, so

$$
\mathrm{N} / \mathrm{n}=(\mathrm{m}+1)^{\mathrm{g}} ; \mathrm{g}=\ln (\mathrm{N} / \mathrm{n}) / \ln (\mathrm{m}+1) \text {. }
$$


We find an implicit relation for $\mathrm{g}$ by substituting $\tau_{1}$ from (10) into $\mathrm{N} / \mathrm{n}$ from (5):

$$
\mathrm{g}=\left[\left(2 \varepsilon_{\mathrm{c}}-\mathrm{m}^{\mathrm{g} / 2} \varepsilon_{\mathrm{d}} / 2\right) / \mathrm{kT}-\ln 4\right] / \ln (\mathrm{m}+1) .
$$

During the carrier lifetime $\tau_{1}$, the number of sites visited is $(m+1)^{g}$. The number of steps taken is $(m+2)^{g}$, which is related to the permittivity in the following way. Each step is influenced by the applied dc field $\mathrm{E}_{0}$ according to a Boltzmann factor. When $\mathrm{E}_{0}$ is first applied, each of the $\mathrm{n}$ carriers per unit volume on the average will change its dipole moment by $\mathrm{p}_{1}$ per diffusion step. After the mean carrier lifetime has elapsed, $(\mathrm{m}+2)^{\mathrm{g}}$ steps have been taken and so one can expect that the limiting polarization is $\mathrm{n}(\mathrm{m}+2)^{\mathrm{g}} \mathrm{p}_{1}=\mathrm{P}_{\mathrm{dc}}$, so that from $\mathrm{D}_{\mathrm{dc}}=\varepsilon_{0} \varepsilon_{\mathrm{s}} \mathrm{E}_{0}=\varepsilon_{0} \varepsilon_{\infty} \mathrm{E}_{0}+\mathrm{P}_{\mathrm{dc}}$ we have

$$
\varepsilon_{\mathrm{s}}=\varepsilon_{\infty}+P_{\mathrm{dc}} / \varepsilon_{0} \mathrm{E}_{0}=\varepsilon_{\infty}+\mathrm{n}(\mathrm{m}+2)^{\mathrm{g}} \mathrm{p}_{1} / \varepsilon_{0} \mathrm{E}_{0}
$$

Here, $p_{1}$ could be estimated from the spontaneous polarization of DRDP, but in this work we will determine it from the measured low-frequency limit of the permittivity, $\varepsilon_{\mathrm{sm}}$, and the known high-frequency permittivity, $\varepsilon_{\infty}$, with the help of Eq. (13):

$$
\mathrm{p}_{1}=\varepsilon_{0} \mathrm{E}_{0}\left(\varepsilon_{\mathrm{sm}}-\varepsilon_{\infty}\right) / \mathrm{n}(\mathrm{m}+2)^{\mathrm{g}} \text {. }
$$

Here, $\varepsilon_{\mathrm{sm}}$ nearly obeys a Curie-Weiss law $\varepsilon_{\mathrm{sm}} \cong \mathrm{C}_{\mathrm{cw}} /\left(\mathrm{T}-\mathrm{T}_{\mathrm{cw}}\right)$ at higher $\mathrm{T}$, but in the deuteron glass regime, $\varepsilon_{\mathrm{sm}}$ saturates at a finite value $\varepsilon_{\mathrm{sm} 0}$ as $\mathrm{T}$ decreases.

\section{FRACTAL GENERATION DISTRIBUTION AND AC PERMITTIVITY}

So far, we assumed that the highest barrier encountered on the annihilation path is $\mathrm{m}^{\mathrm{g} / 2} \varepsilon_{\mathrm{d}} / 2$, with $\mathrm{g}$ an integer for the gth generation fractal diffusion path. For a $\mathrm{dc}$ applied field $\mathrm{E}_{0}$, there is sufficient time for all carriers to cross the highest barriers that they encounter before annihilation, and thus the entire crystal will respond to the field, giving the static contribution $\varepsilon_{\mathrm{s}}-\varepsilon_{\infty}$ for the H-bond-mediated part of the permittivity. For an ac applied field, not all carriers will cross these highest barriers. What is the permittivity contribution of those that do?

For an applied field $\mathrm{E}=\mathrm{E}_{0} \cos \omega \mathrm{t}$, during a positive half-cycle the average field is $2 \mathrm{E}_{0} / \pi$. As an approximation, the field is $\mathrm{E}_{0}$ for a fraction $2 / \pi$ of the half-cycle, and zero for the remainder. In this approximation, the field is on for a time $(2 / \pi)(\pi / \omega)=2 / \omega$. The probability of crossing the barrier per unit time is

$$
(\mathrm{kT} / \mathrm{h}) \exp \left(-\mathrm{m}^{\mathrm{g} / 2} \varepsilon_{\mathrm{d}} / 2 \mathrm{kT}\right)=1 / \tau_{\mathrm{g}},
$$

so the probability of not crossing in the time $2 / \omega$ is $\exp \left(-2 / \omega \tau_{\mathrm{g}}\right)$. Thus the contribution to $\varepsilon(f)-\varepsilon_{\infty}$ from the carriers that cross the highest barriers is (where $f=\omega / 2 \pi$ ) 


$$
\varepsilon(\mathrm{f}, \mathrm{g})-\varepsilon_{\infty}=\left[1-\exp \left(-2 / \omega \tau_{\mathrm{g}}\right)\right]\left(\varepsilon_{\mathrm{s}}-\varepsilon_{\infty}\right) /\left(1+\mathrm{i} \omega \tau_{\mathrm{g}}\right),
$$

where a Debye relaxation contribution is assumed.

What is the contribution of carriers that cross g-1 generation barriers but not $g$ generation barriers? The fraction of such carriers is

$$
\exp \left(-2 / \omega \tau_{\mathrm{g}}\right)\left[1-\exp \left(-2 / \omega \tau_{\mathrm{g}-1}\right)\right]
$$

We must also consider how much polarization results from a g-1 path, compared to a $\mathrm{g}$ path. We saw before that the polarization response is proportional to $(\mathrm{m}+2)^{\mathrm{g}}$, so a g-1 path has only a fraction $1 /(\mathrm{m}+2)$ as much response as a g path. Thus,

$$
\varepsilon(\mathrm{f}, \mathrm{g}-1)-\varepsilon_{\infty}=(\mathrm{m}+2)^{-1} \exp \left(-2 / \omega \tau_{\mathrm{g}}\right)\left[1-\exp \left(-2 / \omega \tau_{\mathrm{g}-1}\right)\right]\left(\varepsilon_{\mathrm{s}}-\varepsilon_{\infty}\right) /\left(1+\mathrm{i} \omega \tau_{\mathrm{g}-1}\right) \text {. }
$$

Here, $\tau_{\mathrm{g}-1}$ is found from Eq. (17) by substituting g-1 for $\mathrm{g}$.

In these expressions, the probabilities $\mathrm{W}(\mathrm{f}, \mathrm{k})$ that the carrier traverses a $\mathrm{k}$ generation fractal path but not a $\mathrm{k}+1$ generation path must add to unity:

$$
\sum_{k=0}^{g} \mathrm{~W}(\mathrm{f}, \mathrm{k})=\sum_{k=0}^{g}\left\{\left[1-\exp \left(-2 / \omega \tau_{\mathrm{k}}\right)\right] \exp \left[\sum_{j=k+1}^{g}\left(-2 / \omega \tau_{\mathrm{j}}\right)\right]\right\}=1,
$$

where we must set $\tau_{0}=0$ to make the sum equal to unity. The permittivity contribution at frequency $\mathrm{f}$ from the $\mathrm{H}$-bond mechanism is

$$
\varepsilon(\mathrm{f})-\varepsilon_{\infty}=\sum_{k=0}^{g}\left[(\mathrm{~m}+2)^{\mathrm{k}-\mathrm{g}} \mathrm{W}(\mathrm{f}, \mathrm{k}) /\left(1+\mathrm{i} \omega \tau_{\mathrm{k}}\right)\right] .
$$

\section{INTEGRAL FORMULATION FOR PERMITTIVITY}

The foregoing integer $\mathrm{g}$ and discrete $\mathrm{k}$ values constitute too coarse a formulation, and integer g only occurs at certain temperatures. The sum in Eq. (19) that sums to unity must go over into an integral, with integrand $\mathrm{W}(\mathrm{f}, \mathrm{k})$, that integrates to unity. For the integral, we can drop the special value $\tau_{0}$ that provided unity value for the sum. For $g$ we use the (generally non-integer) value provided by Eq. (12). To make the integral integrate to unity, the upper limit must be larger than $g$ by an amount $\alpha$.

The details of this calculation will appear elsewhere. We present the calculation outline and results here. For compactness of notation, we introduce the symbols

$$
\begin{aligned}
& \mathrm{A}=2 \pi \mathrm{fh} / \mathrm{kT}, \quad \mathrm{B}=\varepsilon_{\mathrm{d}} / 2 \mathrm{kT}, \quad \mathrm{S}_{\mathrm{j}}=\operatorname{Aexp}\left(\mathrm{m}^{\mathrm{j} / 2} \mathrm{~B}\right), \text { and } \\
& \alpha=\ln \left\{[(\mathrm{m}+2) \mathrm{e}]^{-\mathrm{g}}+\ln [(\mathrm{m}+2) \mathrm{e}]\right\} / \ln [(\mathrm{m}+2) \mathrm{e}]
\end{aligned}
$$

where $\mathrm{e}$ is the base of natural logarithms.

The integral formulation expression for $\mathrm{W}(\mathrm{f}, \mathrm{k})$ is 


$$
\mathrm{W}(\mathrm{f}, \mathrm{k})=\left[1-\exp \left(-2 / \mathrm{S}_{\mathrm{k}}\right)\right] \exp \left[\mathrm{k}-\mathrm{g}+\int_{k}^{g} \exp \left(-2 / \mathrm{S}_{1}\right) \mathrm{dl}\right]
$$

The normalized real part of the permittivity then turns out to be

$$
\left[\varepsilon^{\prime}(\mathrm{f})-\varepsilon_{\infty}\right] /\left(\varepsilon_{\mathrm{s}}-\varepsilon_{\infty}\right)=\int_{0}^{g+\alpha}(\mathrm{m}+2)^{\mathrm{k}-\mathrm{g}} \mathrm{W}(\mathrm{f}, \mathrm{k})\left(1+\mathrm{S}_{k}^{2}\right)^{-1} \mathrm{dk}
$$

The normalized imaginary part of the permittivity, $\varepsilon "(f) /\left(\varepsilon_{s}-\varepsilon_{\infty}\right)$, is given by the same expression as in Eq. (24), except that the integrand is multiplied by the factor $S_{k}$.

\section{CONCLUSIONS AND PLANS}

We will next examine polarization decay in zero field, following constant field $\mathrm{E}$ applied for various times. Fourier transformation of the above ac response expressions, necessary for this calculation, has been done, but numerical and graphical results are not complete. This process will predict what sort of memory effects should occur in the polarization. We will also expand our previous analysis ${ }^{3}$ of NMR phenomena to include effects of the fractal diffusion path.

\section{ACKNOWLEDGEMENT}

This work was supported by NSF Grant DMR-9805272.

\section{REFERENCES}

1. Courtens, E., Phys. Rev. B 33, 2975-2978 (1986).

2. Blinc, R., Zalar, B., Dolinšek, J., Kind, R., Cereghetti, P.M., and Jeitziner, C.A., Proceedings of this

Workshop.

3. Schmidt, V.H., AIP Conf. Proc. 582, Fundamental Physics of Ferroelectrics 2001, H. Krakauer, Ed., p. $165-174$.

4. Slater, J.C., J. Chem. Phys. 9, 16 (1941).

5. Takagi, Y., J. Phys. Soc. Jpn. 3, 273 (1948).

6. Schmidt, V.H., Wang, J.T., and Schnackenberg, P., Jpn. J. Appl. Phys. 24, Supplement 24-2, 944946 (1985). 
Copyright $\odot 2002$ EBSCO Publishing 
Copyright of AIP Conference Proceedings is the property of American Institute of Physics and its content may not be copied or emailed to multiple sites or posted to a listserv without the copyright holder's express written permission. However, users may print, download, or email articles for individual use. 